\title{
Central Line-Associated Bloodstream Infection Prevention by Central Venous Catheter Management Staff in the Surgical Intensive Care Unit
}

\author{
Jin-Uk Choi, M.D.*, Nak-Jun Choi, M.D.*, Suk-Kyung Hong, M.D., Ph.D.*, \\ Tae-Hyun Kim, M.D.*, Min Ae Keum, M.D. ${ }^{\dagger}$, Se Ra Kim ${ }^{\dagger}$, \\ Sun Ju Lee ${ }^{\ddagger}$, Sung-Dae Shin ${ }^{\ddagger}$
}

*Division of Acute Care Surgery, Department of Surgery, Asan Medical Center, University of Ulsan College of Medicine, Seoul, ${ }^{\dagger}$ Department of Surgery, Ulsan University Hospital, University of Ulsan College of Medicine, Ulsan, ${ }^{\dagger}$ Department of Critical Care Nursing, Asan Medical Center, University of Ulsan College of Medicine, Seoul, Korea

Correspondence to:
Nak-Jun Choi, M.D.
Division of Acute Care Surgery,
Department of Surgery, Asan
Medical Center, University of
Ulsan College of Medicine, 88
Olympic-ro 43-gil, Songpa-gu,
Seoul 05505, Korea
Tel: +82-2-3010-1824
Fax: +82-2-474-6701
E-mail: eks7957@naver.com
ORCID:
http://orcid.org/0000-0002-7390-4364
Purpose: Patients in the intensive care unit (ICU) are more susceptible to nosocomial infections, including central line-associated bloodstream infection (CLABSI), surgical site infection, urinary tract infection or ventilator-associated pneumonia. This study is a comparative analysis of how central venous catheter (CVC) management staff affects CLABSI.

Methods: We performed a two-phase review of all patients transferred to the surgical ICU (SICU) from January 2013 to June 2014. CVC management staff was introduced in October 2013. Electronic medical records provided the data for a comparative analysis of incidence rates and risks of CLABSI, as well as the subjects' general characteristics.

Results: This study included 248 patients before the introduction of a CVC management staff member and 196 patients after the introduction. General patient characteristics before and after the CVC management staff was in place did not differ significantly. The CLABSI rate decreased by 4.61 cases/1,000 device days after the introduction (6.26 vs. 1.65; odds ratio, 4.47; $95 \%$ confidence interval, 1.39 14.37; $\mathrm{p}=0.009)$. However, the mortality rate and length of ICU stay did not change after CVC management staff was in place $(12.9 \%$ vs. $10.7 \%$, $p=0.480$; $16.00 \pm 24.89$ vs. $15.87 \pm 18.80, \mathrm{p}=0.954$; respectively).

Conclusion: In this study, the introduction of CVC management staff effectively reduced CLABSI rates in current ICU system. (J Acute Care Surg 2018;8:65-70)

Key Words: Central venous catheters, Infection, Prevention

Received September 29, 2017, Revised October 16, 2017, Accepted October 23, 2017

Copyright $(2018$ by Korean Society of Acute Care Surgery

(c) This is an Open Access article distributed under the terms of the Creative Commons Attribution Non-Commercial License (http://creativecommons.org/licenses/by-nc/4.0) which permits unrestricted non-commercial use, distribution, and reproduction in any medium, provided the original work is properly cited.

ISSN 2288-5862(Print), ISSN 2288-9582(Online)

https://doi.org/10.17479/jacs.2018.8.2.65

\section{Introduction}

The number of patients predisposed to nosocomial infections has been on the rise recently owing to an increase in immunologically compromised cases caused by the increasing trend of aging and patients with a chronic disease as well as administration of anticancer agents or immunosuppressants [1]. Patients in the intensive care unit (ICU) are more susceptible 
to nosocomial infections than those in the general ward and are also predisposed to the risk of contracting an infection through various medical devices. Foley catheters, central venous catheters (CVCs), and ventilators are typical medical devices used in ICUs, and their relevant infections, such as catheter-associated urinary tract infection and central line-associated bloodstream infection (CLABSI), or ventilator-associated pneumonia are the subject of intense focus [2]. A CVC is a device used to administer fluids and pharmacological agents and for hemodynamic observations and hemodialysis. It is commonly used in the management of critically ill patients [3,4]. However, a CVC is an invasive device that may cause mechanical complications, such as pneumothorax, hemothorax, arterial damage, or hematoma as well as bloodstream infections during maintenance of the CVC [5]. The Centers for Disease Control and Prevention (CDC) provide CLABSI prevention guidelines for aseptic insertion and proper CVC management [6]. In this study, a staff member was assigned to take full charge of the CVC management to concur with the CDC's CLABSI guidelines to effectively prevent CLABSI. This study attempts to make comparative analyses of how having a CVC management staff member affects CLABSI.

\section{Methods}

This two-phase retrospective study included 248 patients whose
CVCs had been managed by a nurse or a medical intern before the introduction of a CVC management staff member from January to September 2013 and 196 patients after the introduction of a CVC management staff member from October 2013 to June 2014, commencing the staff member's exclusive CVC management service in October 2013. This study analyzed all patients transferred to the surgical ICU (SICU) for 9 months before and after the introduction of a CVC management staff member. According to the diagnostic criteria for CLABSI, we excluded patients (1) who had been discharged from the ICU within 48 hours and or (2) patients with no CVC or patients from whom a CVC had been removed within 48 hours. A CVC management staff member is the person who effectively manages CVCs for all patients to prevent CLABSI and is responsible for checking the condition of the CVC once a day, changing the dressing regularly, replacing contaminated dressings in compliance with the CLABSI management protocol, and reporting inflammatory reactions of a CVC to the attending physician and removing such a CVC. Electronic medical records were used for obtaining the data and comparatively analyzing incidence rates and risks of CLABSI as well as the subjects' general characteristics (such as sex, age, mortality rate, Acute Physiology and Chronic Health Enquiry (APACHE) II score, duration of ICU stay, length of hospital stay, length of ventilator use, reason for and method of SICU transfer before and after the introduction of a CVC

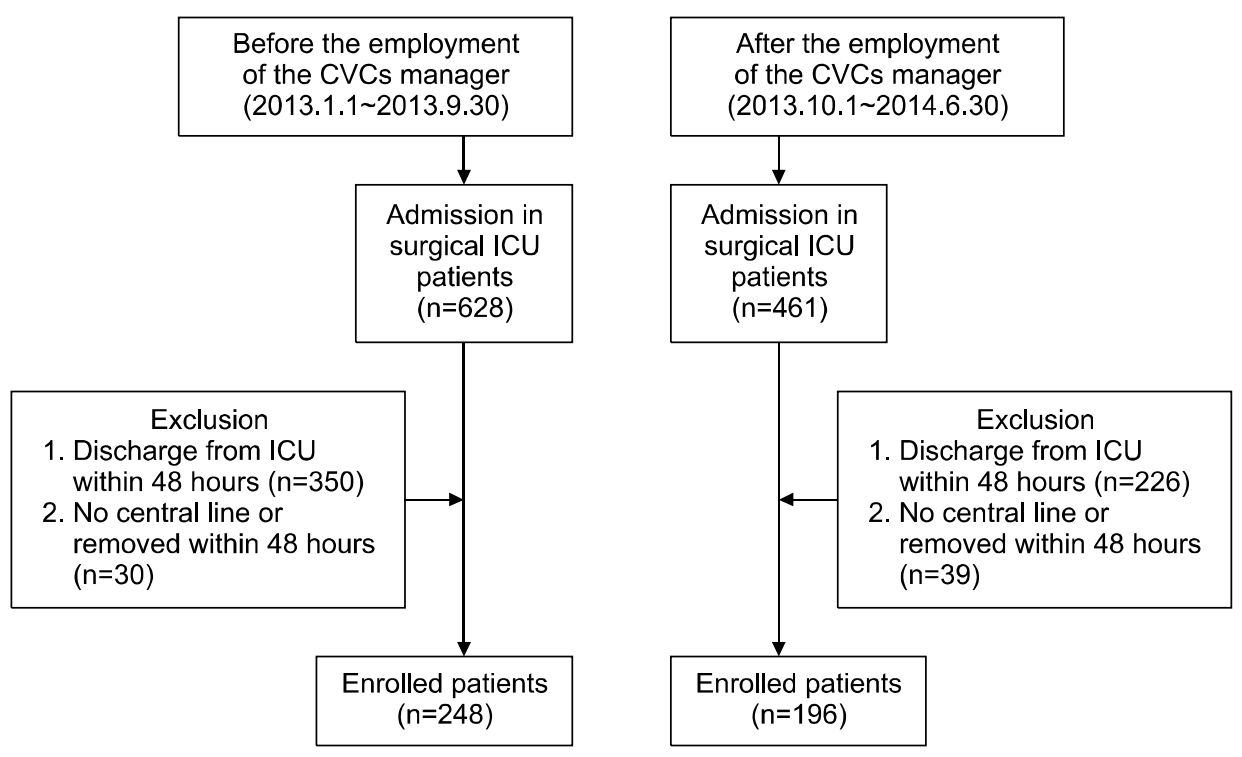

Fig. 1. Flow chart of enrolled patients. CVCs: central venous catheters, ICU: intensive care unit. 
management staff member).

\section{Statistical analyses}

IBM SPSS Statistics ver. 21.0 (IBM Co., Armonk, NY, USA) was used for the statistical analyses. All data were expressed as mean \pm standard deviation or number (\%). Patients' baseline characteristics were compared using Student's t-test for continuous variables and the $\chi^{2}$ test for categorical variables. Logistic regression and univariate analysis was performed to investigate CVC management staff's contribution for CLABSI events and rate. p-value of $<0.05$ was considered to be statistically significant.

This study was approved by Asan Medical Center Institutional Review Board, Seoul, Republic of Korea (IRB no. 2015-0814).

\section{Results}

\section{General characteristics}

This study included 248 patients before the introduction of a

Table 1. Patients' baseline characteristics

\begin{tabular}{lccc}
\hline \multicolumn{1}{c}{ Characteristic } & Pre $(\mathrm{n}=248)$ & Post $(\mathrm{n}=196)$ & p-value \\
\hline Sex (male) & $169(68.1)$ & $136(69.4)$ & 0.779 \\
Age (y) & $60.85 \pm 15.51$ & $63.39 \pm 15.11$ & 0.084 \\
APACHE II score & $21.14 \pm 7.58$ & $21.59 \pm 8.35$ & 0.553 \\
DM & $70(28.2)$ & $58(29.6)$ & 0.752 \\
Smoking & $110(44.4)$ & $99(50.5)$ & 0.197 \\
Length of ICU & $16.00 \pm 24.89$ & $15.87 \pm 18.80$ & 0.954 \\
stay (d) & & & \\
Length of hospital & $55.94 \pm 54.98$ & $53.20 \pm 47.04$ & 0.580 \\
stay (d) & & & \\
Ventilator day & $17.01 \pm 19.93$ & $17.47 \pm 23.24$ & 0.486 \\
Mortality rate & $32(12.9)$ & $21(10.7)$ & 0.480 \\
Reason for ICU admission & & & 0.552 \\
Postoperative care & $118(47.6)$ & $90(45.9)$ & \\
Trauma & $28(11.3)$ & $28(14.3)$ & \\
Septic shock & $26(10.5)$ & $21(10.7)$ & \\
Respiratory failure & $44(11.7)$ & $40(20.4)$ & \\
Other & $32(12.9)$ & $17(8.7)$ & \\
ICU admission route & & & \\
Emergency room & $27(10.9)$ & $26(13.3)$ & \\
General ward & $52(21.0)$ & $33(16.8)$ & \\
Other ICU & $69(27.8)$ & $55(28.1)$ & \\
Operation room & $100(40.3)$ & $82(41.8)$ & \\
\hline
\end{tabular}

Values are presented as number $(\%)$ or mean \pm standard deviation. APACHE II score: Acute Physiology and Chronic Health Enquiry II score, DM: diabetes mellitus, ICU: intensive care unit.
CVC management staff member and 196 patients after the introduction (Fig. 1). The patient population in both periods was predominantly male, with $169(68.1 \%)$ and $136(69.4 \%)$ patients before and after the introduction being men, respectively. There were no statistically significant differences between the two groups in terms of general characteristics, APACHE II scores, diabetes mellitus and smoking status, which can affect CLABSI. Also, there were no statistically significant differences in terms of length of ICU and hospital stay $(16.00 \pm 24.89$ vs. $15.87 \pm 18.80, \mathrm{p}=0.954$; $55.94 \pm 54.98$ vs. $53.20 \pm 47.04, p=0.580$; respectively), length of ventilator use $(17.01 \pm 19.93$ vs. $17.47 \pm 23.24, \mathrm{p}=0.486)$, mortality rate $(12.9 \%$ vs. $10.7 \%, \mathrm{p}=0.480)$, transfer route to the ICU, or the reason for ICU transfer (Table 1).

\section{Pathogens of CLABSI}

Before the introduction 17 CLABSI were reported. Main pathogens were Acinetobacter baumannii, Enterococcus faecium, Staphylococcus epidermidis, and Staphylococcus aureus. After the introduction 4 CLABSI cases were reported and S. epidermidis was main pathogen. Most of pathogens were multidrug-resistant (such as methicillin resistant Staphylococcus aureus, vancomycin resistant enterococcus, carbapenem resistant acinetobacter baumannii and carbapenem resistant pseudomonas aeruginosa) (Table 2).

\section{Central line-associated bloodstream infections}

The mean number of device days for CVCs before the introduction was 2,714 . There were a total of 17 CLABSI cases

Table 2. CLABSI pathogens and CLABSI related mortality

\begin{tabular}{llc}
\hline \multicolumn{1}{c}{ Pathogen } & Pre $(\mathrm{n}=17)$ & Post $(\mathrm{n}=4)$ \\
\hline Staphylococcus epidermidis & $3(17.6)$ & $2(50.0)$ \\
Staphylococcus hemolyticus & $1(5.9)$ & \\
Staphylococcus aureus & $2(11.8)$ & $1(25.0)$ \\
Staphylococcus hominis & $1(5.9)$ & \\
Enterococcus faecium & $3(17.6)$ & \\
Acinetobacter baumannii & $4(23.5)$ & \\
Candida albicans & $1(5.9)$ & $1(25.0)$ \\
Candida tropicalis & & \\
Klebsiella pneumonia & $1(5.9)$ & \\
Pseudomonas aeruginosa & $1(5.9)$ & \\
\hline
\end{tabular}

Values are presented as number (\%).

CLABSI: central line associated blood stream infection. 
during the device days before the introduction, revealing an infection rate of 6.26 cases $/ 1,000$ device days. The mean number of device days for CVCs after the introduction was 2,418. Infection occurred in four cases during the 2,418 device days, showing an infection rate of 1.65 cases $/ 1,000$ device days. The CLABSI rate was decreased by 4.61 cases/1,000 device days after the introduction. The odds ratio was measured as 4.47 (95\% confidence interval, $1.39 \sim 14.37 ; \mathrm{p}=0.009)$. The odds ratio of CLABSI was decreased by $77.6 \%$ after the introduction (Table 3).

\section{CLABSI analysis}

A CLABSI developed in 17 cases before the introduction. The mean duration from the day of CVC insertion to that of confirmed infection was $13.64 \pm 3.75$ days, and there was no case with early infection (the development of an infection within seven days).

With respect to locations, the numbers of cases (and proportions) of CVC insertion in the ICU, angiography unit, emergency department, and operating room were seven (41.2\%), six $(35.3 \%)$, two $(11.8 \%)$, and two $(11.8 \%)$, respectively. The types of CVC included a temporary central line in 16 cases (94.1\%) and a permanent central line in one case $(5.9 \%)$. The sites of
CVC insertion were the internal jugular vein (10 cases, 58.8\%) and the subclavian vein (7 cases, 41.2\%). Regarding insertion positions, a CVC was introduced into the right side in 12 cases $(70.6 \%)$ and the left side in 5 cases $(29.4 \%)$. Among hematogenous infections associated with CVCs, a diagnosis of catheter-related bloodstream infection was made in two cases $(11.8 \%)$.

Hematogenous CLABSI developed in four cases after the introduction. The mean duration from $\mathrm{CVC}$ insertion to confirmed infection was $12.25 \pm 9.94$ days, and there were two cases $(50.0 \%)$ with early infection (the development of an infection within 7 days). Concerning locations, the number of cases (and proportions) of CVC insertion in the ICU, angiography unit, and operating room were two $(50.0 \%)$, one $(25.0 \%)$, and one $(25.0 \%)$, respectively. The site of CVC insertion in these cases (75.0\%) was the internal jugular vein. With respect to insertion position, a CVC was inserted into the right side in four cases. Among hematogenous infections associated with CVCs, a diagnosis of catheter-related bloodstream infection was made in one case $(25.0 \%)$.

The CLABSI rate seven days after CVC insertion, by which the effect of CVC management care can be reflected, was 6.26 before the introduction and 0.83 after the introduction. A

Table 3. Central line-associated bloodstream infection rate attributes

\begin{tabular}{lcccc}
\hline \multicolumn{1}{c}{ Characteristic } & Pre $(\mathrm{n}=248)$ & Post $(\mathrm{n}=196)$ & Odds ratio $(95 \% \mathrm{CI})$ & $\mathrm{p}$-value \\
\hline CLABSI (no. of events) & 17 & 4 & & \\
CVC device days & 2,714 & 2,418 & & \\
CLABSI rate & 6.26 & 1.65 & $4.47(1.39 \sim 14.37)$ & 0.009 \\
\hline
\end{tabular}

CI: confidence interval, CLABSI: central line-associated bloodstream infection, CVC: central venous catheter.

${ }^{\text {a) }}$ CLABSI rate $=$ CLABSI events $/$ CVC days $\times 1,000$.

Table 4. Analysis event of central line-associated bloodstream infections

\begin{tabular}{lccc}
\hline Characteristic & Pre $(\mathrm{n}=17)$ & Post $(\mathrm{n}=4)$ & p-value \\
\hline CLABSI events before 7 days & $0(0)$ & $2(50.0)$ & 0.427 \\
CLABSI rate before 7 days ${ }^{\mathrm{a})}$ & 0 & 0.83 & $<0.001$ \\
CLABSI events after 7 days & $17(100.0)$ & 0.83 & 0.544 \\
CLABSI rate after 7 days a $^{\mathrm{a}}$ & 6.26 & $2(50.0)$ & \\
CLABSI related mortality & $4(23.5)$ & & \\
\hline
\end{tabular}

Values are presented as number (\%) or number only.

CLABSI: central line-associated bloodstream infection.

${ }^{a)}$ CLABSI rate $=$ CLABSI events $/$ CVC days $\times 1,000$.

${ }^{b)}$ CLABSI related mortality $=$ mortality cases due to CLABSI events. 
statistically significant reduction in CLABSI rate between the two groups $(\mathrm{p}<0.001)$ was confirmed.

In total CLABSI event four cases of CLABSI related mortality reported before introduction $(23.5 \%)$ and two cases of related mortality reported after introduction $(50.0 \%)$ but with no statistically significant difference $(\mathrm{p}=0.544)$ (Table 4).

\section{Discussion}

A recent increased concern over nosocomial infections secondary to the use of invasive medical devices has led to the founding of the Korean National Healthcare-Associated Infections Surveillance System (KONIS). KONIS has continuously monitored nosocomial infections since August 2006 [7]. Countries such as Japan and the United States have operated the Japanese Nosocomial Infection Surveillance and the National Nosocomial Infection Surveillance. Each nation has made efforts to monitor medical device-related infections as the infection surveillance data were publicized. CLABSI connotes a confirmation of a hematogenous infection without no other specific cause of blood-borne infection within two days of CVC insertion. The CLABSI rate is the index by which the extent of hematogenous infection caused by a CVC is expressed [8].

According to the KONIS data published in 2012, the mean CLABSI rate in ICUs was $3.01(2.84 \sim 3.19)$ cases/1,000 catheter days, and the mean length of device insertion was $0.52(0.519 \sim$ $0.521)$ days. They reported that $87.1 \%$ of all hematogenous infections were CLABSI cases [9]. The U.S. National Healthcare Safety Network data published in 2013 stated that the mean CLABSI rate in ICUs was $0.8 \sim 1.6$ cases $/ 1,000$ catheter days. This figure was somewhat lower than that of the Republic of Korea [10]. As such, the reason many hospitals get on the bandwagon of investigating and reporting CLABSI data worldwide is in part due to the increased length of ICU stay as well as expanded medical expenditure caused by such infections, which in turn leads to an extended length of treatment and increased economic burden for patients [11-13]. Thus, above all, effective prevention against CLABSI is imperative.

As effective CLABSI prevention, the CDC suggests a checklist with three guiding principles: first, promptly remove unnecessary central lines; second, follow proper insertion practices; and third, handle and maintain central lines and dressings appropriately [6]. On this basis, hand washing practice as a CLABSI prevention activity was vigorously engaged at the outset. Thereafter, CVC maintenance bundles were prepared in an effort to prevent CLABSI. These prevention activities resulted in a CLABSI rate. The fact is that the CLABSI rate has been reduced through these procedures. Nonetheless, an attempt has been made to further decrease CLABSI through effective CVC management. The US CDC has prepared and suggests using a checklist for CVC management and proper dressing.

Notwithstanding, in reality, there are numerous difficulties to implement this management in clinical practice. In cases where a nurse in charge or a medical intern manages CVCs, notwithstanding having a continued training on CVC management, a hospital working environment with rotating duties and shift working of physician and nurse makes it difficult to grasp the status change and constant care of a CVC for each patient. Thus, effective CVC management would not be likely in such a setting. Therefore, it was considered that a CVC management staff member solely in charge of CVCs could be a commendable proposal. A CVC management staff member performs the duties of implementing appropriate dressing practices for CVCs in compliance with the CVC management protocol suggested by the US CDC guidelines. Appropriate CVC dressing practices include maintaining proper sanitation of the hands; replacement of dressings that are wet, contaminated, or improperly attached; and implementing an aseptic technique during replacement of the CVC dressings. The CVC management staff member does not participate in CVC insertions. Nevertheless, it was found that the consistent activities of CVC management could reduce CLABSI. Meanwhile infection management team conduct handwashing training and CVC management checklist monthly in ICU team to reduce CLABSI events.

The prevention and management of CLABSI are one of major axis in care of ICU patients. In this study, introduction of CVC management staff showed decrease in CLABSI rate. Patient's predisposing factors (age, underlying disease, reason for admission) showed no statistically significant difference in two groups (Table 1) and because of low probability of CLABSI event, 
univariate analysis was used to show how CVC management staff contributes to CLABSI events and rate. Despite of this limitation, this study suggests the introduction of CVC management staff was an effective way to reduce CLABSI rate in current ICU system.

\section{Conflicts of Interest}

No potential conflict of interest relevant to this article was reported.

\section{References}

1. Kamboj M, Sepkowitz KA. Nosocomial infections in patients with cancer. Lancet Oncol 2009;10:589-97.

2. Richards MJ, Edwards JR, Culver DH, Gaynes RP. Nosocomial infections in combined medical-surgical intensive care units in the United States. Infect Control Hosp Epidemiol 2000;21:510-5.

3. Maki DG, Mermel LA. Infections due to infusion therapy. In: Bennett JV, Brachman PS, eds. Hospital infections. 4th ed. Philadelphia: Lippincott-Raven; 1998. p. 689-724.

4. Raad II, Hanna HA. Intravascular catheter-related infections: new horizons and recent advances. Arch Intern Med 2002; 162:871-8.

5. McGee DC, Gould MK. Preventing complications of central venous catheterization. N Engl J Med 2003;348:1123-33.

6. O'Grady NP, Alexander M, Burns LA, Dellinger EP, Garland J, Heard SO, et al. Summary of recommendations: guidelines for the prevention of intravascular catheter-related infections. Clin Infect Dis 2011;52:1087-99.
7. Choi JY, Kwak YG, Yoo H, Lee SO, Kim HB, Han SH, et al. Trends in the distribution and antimicrobial susceptibility of causative pathogens of device-associated infection in Korean intensive care units from 2006 to 2013: results from the Korean Nosocomial Infections Surveillance System (KONIS). J Hosp Infect 2016;92:363-71.

8. Horan TC, Andrus M, Dudeck MA. CDC/NHSN surveillance definition of health care-associated infection and criteria for specific types of infections in the acute care setting. Am J Infect Control 2008;36:309-32.

9. Woo JH. Construction and operation of the nationwide surveillance system for nosocomial infections in intensive care units [Internet]. Cheongju: KCDC; 2012 [cited 2013 Apr 3]. Available from: www.cdc.go.kr/CDC/cms/cmsFileDownload. jsp?fid $=28 \&$ cid $=20530 \&$ field Name $=$ attach1\&index $=1$.

10. Dudeck MA, Weiner LM, Allen-Bridson K, Malpiedi PJ, Peterson KD, Pollock DA, et al. National Healthcare Safety Network (NHSN) report, data summary for 2012, device-associated module. Am J Infect Control 2013;41:1148-66.

11. Blot SI, Depuydt P, Annemans L, Benoit D, Hoste E, De Waele JJ, et al. Clinical and economic outcomes in critically ill patients with nosocomial catheter-related bloodstream infections. Clin Infect Dis 2005;41:1591-8.

12. Hidron AI, Edwards JR, Patel J, Horan TC, Sievert DM, Pollock DA, et al. NHSN annual update: antimicrobial-resistant pathogens associated with healthcare-associated infections: annual summary of data reported to the National Healthcare Safety Network at the Centers for Disease Control and Prevention, 2006-2007. Infect Control Hosp Epidemiol 2008; 29:996-1011.

13. Umscheid CA, Mitchell MD, Doshi JA, Agarwal R, Williams $\mathrm{K}$, Brennan PJ. Estimating the proportion of healthcare associated infections that are reasonably preventable and the related mortality and costs. Infect Control Hosp Epidemiol 2011;32:101-14. 